\title{
Aufsatz
}

Karl-Heinz Kamp*

\section{Das strategische Langfrist-Problem der NATO}

https://doi.org/10.1515/sirius-2019-2003

Zusammenfassung: Siebzig Jahre nach ihrer Gründung ist die NATO in guter Verfassung und gilt mit Recht als die erfolgreichste Sicherheitsorganisation in der neueren Geschichte. Mittel- und langfristig sieht sie sich allerdings einer existenziellen Herausforderung ausgesetzt, da es immer schwerer werden wird, ihre Relevanz für die Vereinigten Staaten als Bündnisvormacht zu bewahren. Dieses Problem ergibt sich weniger aus der bündnisfeindlichen Politik Donald Trumps, sondern folgt aus der sich abzeichnenden geostrategischen Re-Orientierung der USA weg von Russland und hin zu China. In einer möglichen bipolaren Welt mit China und USA als Konkurrenten wird auch die NATO sich geographisch stärker in den asiatischpazifischen Raum orientieren müssen, wenn sie nicht dramatisch an Bedeutung verlieren will.

Schlüsselbegriffe: NATO, USA, Allianzen, bipolare Welt, Schwerpunktverlagerung nach Asien

\begin{abstract}
Seven decades after it was established, the North Atlantic Alliance is doing fairly well and fully deserves being described as the most successful security organization in modern history. In the long term though, NATO faces an almost existential problem, as it will be difficult to maintain its relevance for the United States as the dominant power within the Alliance. This will be less a result Donald Trump destructive policies than of the geostrategic reorientation of the US away from Russia and towards China. NATO will also have to fundamentally alter its geographic orientation towards the Asia-Pacific region in order to avoid falling into oblivion.
\end{abstract}

Keywords: NATO, USA, military alliances, bipolar world, pivot to Asia

*Kontakt: Dr. Karl-Heinz Kamp, Präsident der Bundesakademie für Sicherheitspolitik, Berlin; und Mitherausgeber von SIRIUS, E-Mail: karl-heinz.kamp@baks.bund.de

\section{Einleitung}

$\mathrm{Zu}$ ihrem siebzigsten Jubiläum präsentiert sich die so oft totgesagte Nordatlantische Allianz in erstaunlich guter Verfassung. Durch eine ständige Anpassung an neue sicherheitspolitische Anforderungen hat sie auf die Veränderungen der vergangenen Jahrzehnte reagiert und stets ihre eigene Relevanz bewahrt. Sie hat zum Ende des OstWest-Konflikts ebenso beigetragen wie zum Aufbau einer gesamteuropäischen Sicherheitsordnung nach dem Ende des Kalten Krieges. Als diese 2014 von einem revanchistischen Russland eingerissen wurde, fand die NATO rasch zu ihrer klassischen Rolle als Garant der Sicherheit und territorialen Integrität ihrer Mitglieder gerade in Osteuropa zurück. Darüber hinaus widmet sie sich den Gefahren, denen sich die südlichen Mitgliedsstaaten ausgesetzt sehen. Selbst das merkwürdige Agieren eines bündnisfeindlichen Präsidenten im Weißen Haus hat die NATO bislang heil überstanden, auch, weil die überwiegende Mehrheit amerikanischer Entscheidungsträger von der Relevanz des Bündnisses überzeugt ist.

So weit, so gut. Langfristig sieht sich die NATO allerdings einem existenziellen Problem gegenüber, das weder in den aktuellen transatlantischen Spannungen noch in der Sprunghaftigkeit von Präsident Donald Trump begründet ist. Es erwächst stattdessen aus den grundlegenden geostrategischen Veränderungen der kommenden Jahre. Mit einer stetig zunehmenden Rolle Chinas bei einer gleichzeitig abnehmenden Bedeutung Russlands werden sich die USA weit stärker als bisher dem asiatischpazifischen Raum zuwenden und Europa trotz aller Treueschwüre eher hinten anstellen. Will die NATO nicht einen großen Teil ihrer Existenzberechtigung verlieren, wird sie ihre geografische Orientierung ebenfalls deutlich ändern und ausweiten müssen. 


\section{Die erfolgreichste Sicherheits- allianz in der Geschichte}

Bis zum „Wendejahr“ 2014 haben Beobachter der NATO deren Geschichte gerne in drei Phasen eingeteilt. ${ }^{1}$ In ihrer ersten Phase, die von ihrer Gründung im Jahr 1949 bis zum Fall der Berliner Mauer 1989 reichte, war das Bündnis vor allem ein Instrument westlicher Selbstbestimmung und Verteidigung. In ihrer zweiten Phase, die sich bis über die Jahrtausendwende erstreckte, füllte die NATO das Machtvakuum der untergegangenen Sowjetunion aus und leistete Hilfe bei der Demokratisierung Osteuropas. In ihrer dritten Phase, die am 11. September 2001 mit dem Zusammenbruch der Twin Towers in New York einsetzte, entwickelte sich die Allianz zu einem globalen Sicherheitsakteur, der sich um die Stabilisierung von Krisenregionen weitab der eigenen Bündnisgrenzen bemühte. Der Ausspruch des damaligen Verteidigungsministers Struck, dass deutsche Sicherheit auch am Hindukusch verteidigt werde, war prägend für diese Epoche. Mit Moskaus Annexion der Krim und den russischen Truppen in der Ostukraine im Jahr 2014 begann die vierte und derzeitige Phase. Seither befindet sich die NATO abermals in der Artikel-5-Welt, in einem sicherheitspolitischen Umfeld also, in dem das Sicherheitsversprechen des Artikels 5 des Washingtoner Vertrages wieder mit glaubwürdigen militärischen Fähigkeiten untermauert sein muss. Im Zentrum der Allianz steht nicht mehr das internationale Krisenmanagement außerhalb ihrer Grenzen, sondern vor allem die Landes- und Bündnisverteidigung zum Schutz des Bündnisgebiets.

Die NATO reagierte außergewöhnlich rasch auf die russische Aggressionspolitik - vermutlich durchgreifender, als Präsident Putin dies erwartet hatte. Mit den Entscheidungen der Gipfeltreffen in Wales 2014 und Warschau 2016 stellte sich das Bündnis auf die neuen Erfordernisse der Artikel-5-Welt ein und erhöhte die Schlagkraft ihrer militärischen Fähigkeiten. Die bereits 2002 aufgestellte NATO Response Force (NRF) wurde auf fast 40.000 Soldaten erweitert und um eine 5.000 Mann starke schnelle Eingreiftruppe (Very High Readiness Joint Task Force) ergänzt. Diese Task Force, kurz VJTF, deren Kernbestandteil im laufenden Jahr durch die Bundeswehr gestellt wird, ist in ständiger Bereitschaft und kann in wenigen Tagen in Marsch gesetzt werden. Unter dem Schlagwort Enhanced Forward Presence (EFP) stehen seit 2017 vier gefechtsbereite Bataillone in Estland, Lettland, Litauen und Polen zur Verfügung - jedes von einem der vier großen NATO-

1 Rühle 2006.
Mitglieder USA, Kanada, Großbritannien und Deutschland bereitgestellt und in die Truppen der Gastländer integriert. Zusätzlich zu den Truppenstationierungen hat die NATO die Zahl der militärischen Übungen erhöht und an realistische Szenarien angepasst sowie neue Verteidigungspläne entwickelt. Auch wurden die Entscheidungsprozesse in der NATO gestrafft, sodass ein Bündnisbeschluss zum Einsatz der Schnellen Eingreiftruppe innerhalb von acht bis zwölf Stunden getroffen werden kann. ${ }^{2}$

Selbst bei der nuklearen Abschreckung, einem Teil des NATO-Dispositivs, das in vielen Mitgliedsländern der Kritik ausgesetzt ist, wurden wesentliche Verbesserungen realisiert. Dies zeigte sich zunächst in einem neuen nuklearen „Mindset“ in allen NATO-Ländern: Man erzielte Einigkeit über die von Russland ausgehenden nuklearen Gefahren und vergegenwärtigte sich, dass die NATO eine „nukleare Allianz“ ist und bleibt. Das war keinesfalls selbstverständlich, wenn man überlegt, dass vor einem knappen Jahrzehnt der damalige deutsche Außenminister Guido Westerwelle den Abzug aller amerikanischen Atomwaffen von deutschem Boden gefordert und dies sogar in den Koalitionsvertrag von CDU/CSU und FDP eingebracht hatte.

Im Bereich der nuklearen Waffen und Trägersysteme wurden die Reaktionszeiten der nuklearfähigen Flugzeuge, die im Extremfall die in Europa stationierten amerikanischen Kernwaffen transportieren würden, erheblich verkürzt. Das dient vor allem dazu, den nuklearen Bereitschaftsgrad der NATO dem ihrer konventionellen Eingreiftruppen zumindest anzunähern, um so eine schlüssige Gesamtabschreckung zu schaffen. Die US-Kernwaffen selbst unterliegen einem bereits unter Präsident Obama begonnenen grundlegenden Modernisierungsprozess, der in einigen Jahren abgeschlossen sein wird. Auch im nuklearen Bereich wurden die Übungen deutlich erhöht, um durch die Demonstration militärischer Bereitschaft ein starkes politisches Abschreckungssignal an Russland zu senden. Schließlich tragen mehr nicht-nukleare NATO-Staaten zur glaubwürdigen nuklearen Abschreckung bei, indem sie einen (hypothetischen) Einsatz von Kernwaffen mit konventionellen Maßnahmen (Luftbetankung, Bekämpfung gegnerischer Luftabwehr) unterstützen würden. Auch diese im NATO-Jargon „SNOWCAT“ (Support for Nuclear Operations with Conventional Air Tactics) genannten Maßnahmen verdeutlichen die Einigkeit der Allianz in der Nuklearfrage und verstärken damit die Abschreckung insgesamt.

Darüber hinaus kommt die NATO dem Drängen der südlichen Mitgliedsländer nach und kümmert sich im Rahmen

2 Zu weiteren Einzelheiten siehe Brauss 2018. 


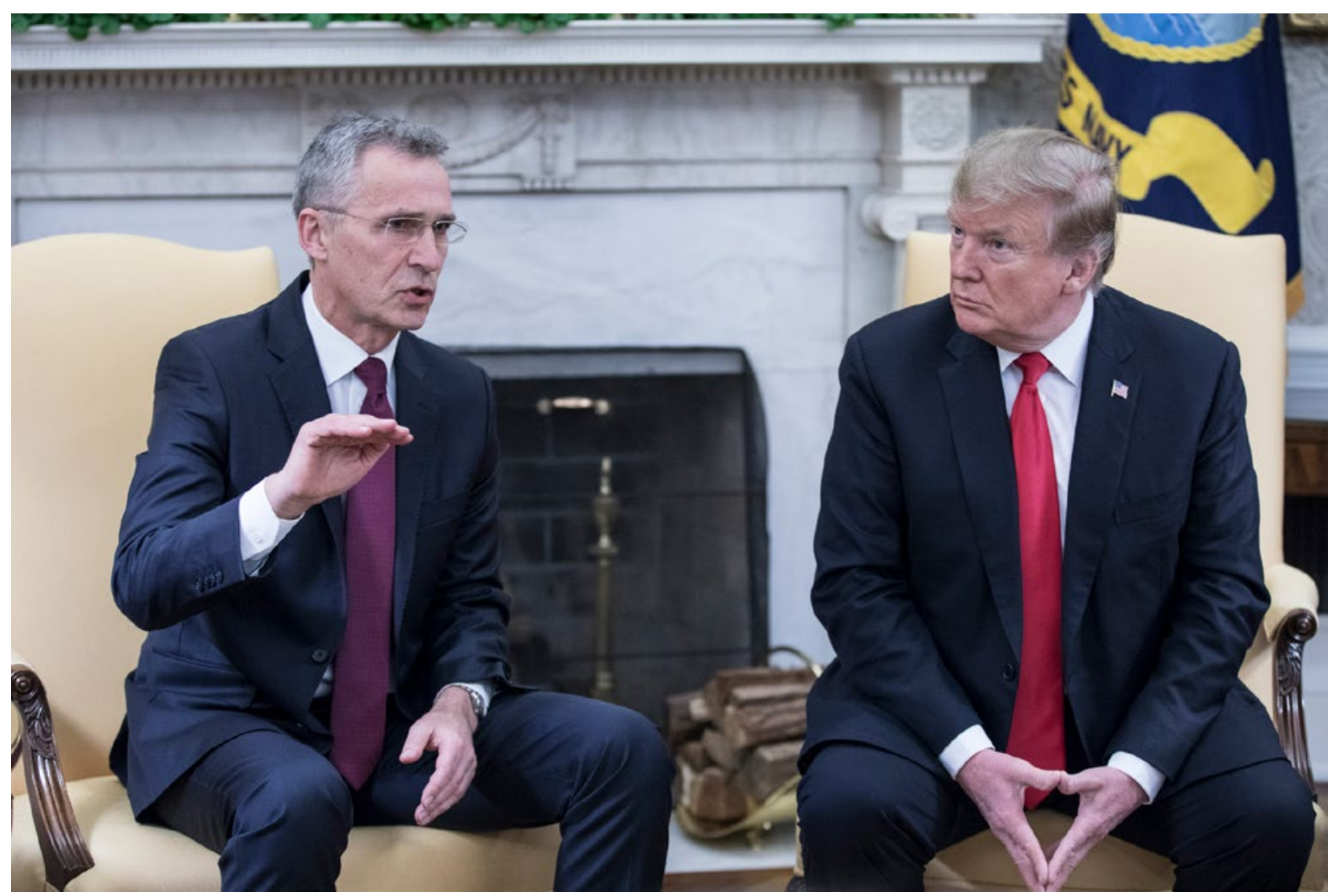

Abb. 1: NATO Generalsekretär Stoltenberg informiert US-Präsident Trump über das Außenministertreffen aus Anlass des 70-jährigen Bestehens der Allianz

ihrer Möglichkeiten um die Sicherheitsgefährdungen aus südlicher und südöstlicher Richtung. Wenn auch der von der Allianz gebrauchte Begriff des „360 Grad-Ansatzes“ etwas übertrieben scheint, so hat die NATO dennoch ihr Präsenz im Schwarzen Meer ausgebaut und unternimmt Schritte gegenüber der globalen Gefahr des Terrorismus.

\section{Amerika bleibt (noch) engagiert}

Dass die NATO den Nimbus als erfolgreichste Sicherheitsallianz der Geschichte erreichen konnte, hing ohne Zweifel ganz wesentlich vom Engagement der USA über sieben Jahrzehnte ab. Somit waren die USA nicht nur die „indispensable nation“ auf der Weltbühne, wie es die ehemalige US-Außenministerin Madeleine Albright schon Ende der neunziger Jahre formulierte, sondern auch der unverzichtbare Sicherheitsgarant Europas. Bemerkenswert ist, dass das amerikanische Engagement in der NATO auch nach der Wahl von Präsiden Donald Trump aufrechterhalten wurde.
Das mag überraschen, ist doch die Verachtung des derzeitigen Präsidenten für multilaterale Institutionen im Allgemeinen und für die NATO im Besonderen deutlich zu spüren. Donald Trump lässt keine Gelegenheit ungenutzt, seine Geringschätzung gerade gegenüber den europäischen Verbündeten öffentlich kundzutun. Seine Auftritte auf NATO-Gipfeltreffen sind mittlerweile gefürchtet und haben zu Überlegungen geführt, Treffen der Staats- und Regierungschefs der Allianz weitgehend einzuschränken. Auch zum 70. Jubiläum war ursprünglich - anders als bei früheren runden Geburtstagen - nur ein Treffen in Washington auf der Ebene der Außenminister geplant. Nur dem heftigen Drängen Großbritanniens, das als Folge des anstehenden EU-Austritts nach einer besonderen Rolle in der NATO sucht, ist es zu verdanken, dass es im Dezember 2019 zu einem sogenannten „High Level Meeting“ in London kommt, bei dem auch Donald Trump anwesend sein dürfte.

Bei genauerer Betrachtung belegen die morgendlichen Twitter-Botschaften gegen die amerikanischen Allianzverpflichtungen eher die völlige politische Ahnungslosigkeit des Präsidenten als ein Abrücken der USA von 
der NATO. De facto hat sich das amerikanische militärische Engagement in Osteuropa seit dem Amtsantritt Trumps sogar deutlich verstärkt. Seit 2014 haben die USA in den sogenannten drei „C“ - Cash, Capabilities und Contributions - ganz Erhebliches geleistet. Mit Blick auf finanziellen Beiträge (Cash) hat die Trump-Administration die Finanzhilfen für osteuropäische NATO-Mitglieder vervielfacht. Unmittelbar nach der russischen Aggression gegen die Ukraine hatte die Obama-Administration im Rahmen einer „European Reassurance Initiative“ (ERI) eine Milliarde USD für 2015 bereitgestellt, um Osteuropa militärisch zu stärken. 2016 - ebenfalls noch unter Obama sank dieser Betrag auf 800000 USD. 2017 vervierfachte die gerade ins Amt gekommene Trump-Administration diesen Betrag auf 3,4 Milliarden USD und erhöhte nochmals auf 4,7 Milliarden für das Folgejahr. Für 2019 sind sogar 6,5 Milliarden USD für das Programm veranschlagt, das mittlerweile in „European Deterrene Initiative“ (EDI) umbenannt wurde. ${ }^{3}$

Mit Blick auf Fähigkeiten und Verpflichtungen (Capabilites und Commitments) hat Washington sein Engagement für die NATO belegt, indem es sich massiv in Osteuropa engagiert. Kampfbrigaden werden rotationsweise nach Europa verlegt, Waffen und Munition werden in größeren Mengen eingelagert und die militärische Infrastruktur (Flughäfen, Stützpunkte) wird verbessert. Auch wurden die maritimen Fähigkeiten im Nordmeer - und hier insbesondere die U-Boot-Abwehr - verstärkt. All diese Fähigkeiten werden durch regelmäßige Übungen leistungsfähig gehalten, wodurch auch ein deutliches Signal der Entschlossenheit an Russland gesendet wird.

Im Dezember 2018 trat Verteidigungsminister Mattis, der als Verteidiger des weltpolitischen Engagements der USA galt, zwar nicht völlig überraschend, aber dennoch zu diesem Zeitpunkt unerwartet, von seinem Amt zurück. Das Ausscheiden eines der letzten „Erwachsenen“ im Kabinett Trump löste in der Allianz erhebliche Besorgnis aus, schien damit doch das letzte Bollwerk gegen Trumps Drohung vom Austritt Amerikas aus der NATO gefallen zu sein. Allerdings unterschätzen solche Befürchtungen das hohe Ansehen, das die NATO parteiübergreifend im amerikanischen Kongress genießt. Gerade um den Präsidenten von der Umsetzung unbedachter isolationistischer Reflexe abzuhalten, wird in Beschlüssen des Kongresses die Rolle der Atlantischen Allianz gerade zum Schutz vor russischem Abenteurertum in Osteuropa immer wieder betont. So wird die Freigabe des US-Verteidigungsbudgets stets an das Fortbestehen des amerikanischen Engagements in der NATO gebunden. Der National Defense Authorization

3 Towell/Kazlauskas 2018.
Act (NDAA) betont auch im Jahr 2019 wieder, dass es die Politik der USA sei ,....to fulfill the ironclad commitment of the United States to its obligations under the North Atlantic Treaty ... as part of a broader, long-term strategy backed by all elements of United States national power to deter and, if necessary, defeat Russian aggression". ${ }^{4}$ Solche rechtlichen Regelungen können Präsident Trump kaum von Kurzschluss-Aktionen, wie dem angekündigten (und später relativierten) Truppenabzug aus Syrien oder Afghanistan abhalten. Sie verhindern aber, dass der Präsident seinem antieuropäischen Reflex folgend - versucht, die transatlantischen Sicherheitsverbindungen dauerhaft $\mathrm{zu}$ kappen.

\section{Der strategische Stolperstein für das Bündnis}

Ungeachtet der transatlantischen Querelen, des Streits um ausreichend hohe Verteidigungshaushalte oder der Differenzen zwischen europäischen Mitgliedsländern steht die NATO heute besser da als vor Russlands Schwenk zur Bedrohungspolitik gegenüber seinen Nachbarn - nämlich militärisch stärker und politisch erstaunlich geeint. Allerdings kann dieser positive Befund nicht verdecken, dass das Bündnis längerfristig mit einem existenziellen Problem konfrontiert ist, das weniger mit der Sprunghaftigkeit Donald Trumps zu tun hat, sondern sich aus der sich verändernden strategischen Weltsicht der USA ergibt.

Wie die Haushaltsgesetze des Kongresses (etwa der NDAA) oder der massive amerikanische Fähigkeitsaufbau in Osteuropa zeigen, ergibt sich Washingtons breite Unterstützung für die NATO vor allem aus der Sorge vor der aggressiven Politik Moskaus. Russland wird als revanchistischer Gegner wahrgenommen, der offenbar bereit ist, zur Wahrung des eigenen Machtanspruchs internationales Recht zu brechen und die mühsam aufgebaute europäische Sicherheitsordnung zu zerstören. Weil Russland aber weder politisch noch wirtschaftlich oder gar militärisch auf Augenhöhe mit den USA agieren kann, greift es auf jene Instrumente zurück, die Autokratien gegenüber Demokratien zur Verfügung stehen: Desinformation und Propaganda, Cyber-Angriffe, Trolle oder Wahlbeeinflussung. Moskau nutzt seine begrenzten Mittel zur Destabilisierung der Ukraine, zum Versuch der Spaltung der NATO oder zur Stützung der Regime in Syrien oder

4 US Congress: John S. McCain National Defense Authorization Act for fiscal year 2019, Section 1248, https://www.congress.gov/bill/115thcongress/house-bill/5515/text. 
Venezuela - alles Schritte, die sich eindeutig gegen die Interessen der USA richten.

Gleichzeitig wird Russland in Amerika und Europa überwiegend als absteigende Macht angesehen, die weit von der einstigen vermeintlichen Größe der Sowjetunion entfernt ist. Das Land hat in den letzten drei Jahrzehnten entscheidende Schritte zur wirtschaftlichen, politischen und gesellschaftlichen Modernisierung verpasst und auch die militärische Modernisierung ist aufgrund begrenzter Mittel nur in Teilen durchgeführt worden. Das russische Brutto-Inlandsprodukt ist deutlich kleiner als etwa das von Italien und das Land hat nur zwei wettbewerbsfähige Güter auf dem Weltmarkt: Waffen und fossile Energieträger. Das Wirtschaftssystem ist hochgradig korrupt und basiert in großen Teilen auf der Verteilung der Öl- und Gaseinkünfte anstatt auf Rechtssicherheit und Innovation. Damit ist man für die nächste Welle der industriellen Revolution - Industrie 4.0 und Digitalisierung - nicht gewappnet und wird im internationalen Vergleich weiter zurückfallen. Konstant niedrige Energiepreise durch geringeren Konsum und größeres Angebot (Fracking) dürften diesen Abwärtstrend weiter befördern.

Selbst der Verteidigungshaushalt musste im letzten Jahr gekürzt werden und die oft von Präsident Putin medienwirksam in Szene gesetzten Waffenschauen erweisen sich zumeist als Propagandaaktionen: Nur ein Bruchteil der meist als Wunderwaffen präsentierten Systeme kommen wirklich bei den Streitkräften an. Zwar bleiben russische Streitkräfte eine Bedrohung für die unmittelbaren Nachbarn, sie büßen ihre Fähigkeit zu globalstrategischem Handeln aber immer weiter ein.

Folglich wird Russland mit einer im Vergleich immer noch großen Armee, einem großen Atomwaffen-Arsenal und dem permanenten Sitz im Sicherheitsrat der Vereinten Nationen aus amerikanischer Sicht stets ein erhebliches internationales „Störpotenzial“ behalten. Moskau wird aber immer weniger in der Lage sein, internationale Politik entscheidend in seinem Sinne zu gestalten

Im krassen Gegensatz dazu wird China, dessen atemberaubende wirtschaftliche Entwicklung sich zunehmend in höheren Militärausgaben und damit stärkeren Streitkräften niederschlägt, als aufsteigende Macht wahrgenommen. Parallel dazu tritt China selbstbewusst auf der Weltbühne auf und betreibt mit gigantischen Wirtschafts- und Infrastrukturprojekten wie der Seidenstraßen-Initiative effektive Macht- und Interessenpolitik. Man muss China nicht einmal aggressive oder imperiale Motive unterstellen - es ist der schiere Machtzuwachs, der Besorgnis auslöst. Dadurch gerät China nämlich in die Position, auf Augenhöhe mit den USA zu agieren und nicht nur Amerikas Rolle in der Pazifikregion herauszufor- dern, sondern die als ungerecht empfundene „westliche“ Weltordnung langfristig durch ein chinesisch dominiertes Modell zu ersetzen.

Die generelle Einschätzung, dass Russland sich im Niedergang und China sich im Aufstieg befindet, wird auf beiden Seiten des Atlantiks im Wesentlichen geteilt. Mehr noch, es besteht ein breiter Konsens, dass beide Trends in ihrer Intensität oder Geschwindigkeit zwar schwanken mögen, sie aber letztlich unumkehrbar sind. Dennoch haben sich die NATO-Mitglieder bislang kaum mit den Folgen beschäftigt, die sich aus dieser Entwicklung für die NATO insgesamt und für die europäischen Mitgliedsstaaten im Besonderen ergeben werden.

\section{Europa verliert an Bedeutung}

Wenn Russland aus amerikanischer Sicht längerfristig nicht mehr als globalstrategische Gefahr, sondern als regionales Problem wahrgenommen wird, das mit begrenzten Mitteln eingehegt werden kann, dann sinkt die Bedeutung Europas als „unsinkbarer Flugzeugträger“ insgesamt. Setzt sich der Abwärtstrend Russlands weiter fort, so könnte Washington in fünf oder zehn Jahren zu der Einschätzung gelangen, dass ein russischer Angriff auf seine Nachbarn mit einer begrenzten Anzahl gut ausgestatteter amerikanischer Kampfverbände in wenigen osteuropäischen Schlüsselstaaten sowie einigen maritimen Fähigkeiten im hohen Norden abgeschreckt werden könne. Die NATO insgesamt und gerade die geografisch entfernt von Russland liegenden NATO-Mitglieder verlören erheblich an Relevanz.

Selbst für die Abschreckung der nuklearen Fähigkeiten eines sich im Niedergang befindlichen Russland würde es nicht mehr der derzeitigen NATO-Stationierungsländer für amerikanische Kernwaffen bedürfen. ${ }^{5}$ Die USA verfügen über rund 6.800 Atomsprengköpfe, von denen etwa 4.000 aktiv sind. Darüber hinaus beabsichtigt die amerikanische Regierung laut ihrem „Nuclear Posture Review“ von 2018, besonders kleine, also mit geringer Sprengkraft versehene seegestützte Systeme zu entwickeln, die speziell der Gefährdung durch Russland gewidmet sind. Damit verfügen die USA über ein breites Spektrum von Atomwaffen zu Land, in der Luft und auf See und sind nicht auf den Abschreckungswert ihrer wenigen in Europa gelagerten Atombomben angewiesen. Das gilt umso mehr,

5 Eine sehr kleine Anzahl von amerikanischen Atombomben sind derzeit in Deutschland, Niederlanden, Belgien und Italien stationiert. In der Türkei gibt es zumindest entsprechende Lagerstätten für diese Waffen. Ob sie derzeit befüllt sind, ist unklar. 
als diese Waffen ohnehin einen stärker allianzpolitischen Wert haben als einen militärischen.

Zwar würde die NATO - ungeachtet gelegentlicher Twitter-Meldungen von Präsident Trump - nicht formell aufgelöst werden, sie würde aber immer weiter an Bedeutung einbüßen, je deutlicher der machtpolitische Abstieg Russlands sichtbar würde. Washington könnte bilateral mit den europäischen Ländern verkehren, die politisch oder für die Stationierung von Streitkräften wichtig sind, ohne sich weiter um einen mühsamen Konsens mit allen 29 NATO-Mitgliedsländern mühen zu müssen. Einige der östlichen NATO-Staaten bemühen sich ohnehin sehr aktiv um bilaterale Abkommen mit den Vereinigten Staaten, da ihnen im Krisenfall die amerikanischen Sicherheitsgarantien glaubhafter erscheinen als die Solidarität ihrer europäischen Nachbarn. Ein derart begrenztes US-Engagement in Europa würde es Washington auch erlauben, militärische Mittel von Europa in den asiatisch-pazifischen Raum umzuschichten, um sich noch stärker gegen Chinas Anspruch einer regionalen und langfristig vielleicht sogar globalen Dominanz zu stellen. Damit würden die USA konsequent den „Pivot to Asia“ umsetzen, der von Präsident Obama zwar angekündigt aber nie vollständig realisiert wurde. ${ }^{6}$

Es ist aus dieser Perspektive sogar wahrscheinlich, dass die USA auch ihr Engagement im Nahen und Mittleren Osten weiter einschränken werden, um Ressourcen für die Auseinandersetzung im asiatisch-pazifischen Raum freizumachen. Dieser Trend zeigt sich bereits heute und wird vor allem von einer immer geringeren amerikanischen Energieabhängigkeit und einem deutlichen „Engagement-Fatigue“ angesichts der kaum zu lösenden Stabilitätsprobleme in dieser Region gespeist.

Folgt man dem Gedankengang eines neuen chinesisch-amerikanischen Bilateralismus, dann wird die NATO ihre Relevanz für die USA nur erhalten können, wenn sie langfristig einen signifikanten Beitrag zur Einhegung chinesischer Machtansprüche leistet. Eine solche NATO, die zu verhindern hilft, dass die liberale „westliche“ Weltordnung durch eine chinesische Variante ersetzt wird, wäre nicht nur für die USA hilfreich, sondern auch für Europa selbst. Das gilt umso mehr, als die Allianz seit Jahren enge partnerschaftliche Beziehungen mit ,westlichen“ Ländern in der Pazifikregion - wie Australien, Neuseeland, Japan oder Südkorea - aufgebaut hat.

6 Siehe Binnendijk 2014.

\section{Die NATO in Asien?}

Eine Hinwendung der Nordatlantischen Allianz in Richtung Asien könnte sich in mehreren Stufen und Intensitäten gestalten. Der erste Schritt wäre, dass die NATO mehr Interesse an der Region zeigen und auch als Allianz die Entwicklungen im asiatisch-pazifischen Raum zur Kenntnis nehmen würde. Dies ist überfällig, da NATO-Mitglieder gezwungen sein könnten, in einer ernsten Krise zumindest Position für oder gegen eine der Konfliktparteien zu ergreifen. Eine solche Parteinahme kann nur auf der Basis von hinreichenden Kenntnissen über die Region erfolgen. Bemerkenswert ist, dass mittlerweile einige NATO-Staaten versuchen, das Thema „China“ auf die Agenden der entsprechenden Gremien - wie etwa die Treffen der Außenund Verteidigungsminister - zu heben. Als sichtbarer Ausdruck dieses Interesses könnte die NATO Verbindungsbüros, sogenannte Liaison Offices, in Japan, Australien oder Südkorea einrichten.

Ein zweiter Schritt würde eine deutlich größere Bereitschaft Europas zu einer fairen Lastenteilung mit den USA hinsichtlich Asien erfordern. Wenn nur die USA über die militärischen Fähigkeiten zur globalen Machtprojektion verfügen, dann müssten sich künftig die Europäer in der NATO militärisch stärker in ihren Nachbarregionen engagieren - etwa vor den Küsten Afrikas oder im Indischen Ozean. Die dort stationierten amerikanischen Einheiten würden entlastet und stünden für Operationen in weiter entfernt liegenden Regionen zur Verfügung.

Langfristig werden die großen europäischen Staaten allerdings, sofern sich der chinesisch-amerikanische Bilateralismus realisiert, nicht umhinkommen, in einem dritten Schritt ihrerseits Fähigkeiten zur weitreichenden Machtprojektion vor allem im maritimen Bereich aufzubauen. Das gilt nicht nur aus der Perspektive der NATO, sondern auch aus der Sicht der EU, wenn diese ihrem eigenen Anspruch des „global Player“ gerecht werden will.

Die Vorstellung, dass eine künftige NATO die Bekämpfung der Gefahren im asiatisch-pazifischen Raum als eine ihrer Kernaufgaben ansieht, mag aus heutiger Sicht für viele Bündnismitglieder unrealistisch scheinen. Die meisten Europäer haben derzeit Schwierigkeiten genug, ausreichende Fähigkeiten für die Landes- und Bündnisverteidigung aufzubauen und zeigen nur eine geringe Bereitschaft, sich auf neue Bedrohungen einzustellen. Allerdings hat sich bereits in der Vergangenheit gezeigt, wie schnell sich eine internationale Lage ändern kann und wie rasch die NATO in der Lage sein muss, darauf zu reagieren. Über Jahrzehnte hielten die meisten NATOStaaten an dem Grundsatz fest, sich nie „Out of Area“, also jenseits der Bündnisgrenzen, engagieren zu wollen. Selbst 
nachdem dieser Grundsatz durch das Eingreifen der NATO auf dem Balkan aufgeweicht wurde, hätte sich niemand in Europa vorstellen können, einmal die eigenen Sicherheitsinteressen im 5.500 Kilometer entfernten Afghanistan zu verteidigen. Genau das geschah buchstäblich über Nacht als die Katastrophe des 11. September 2001 die Weltlage fundamental änderte.

Die NATO passte sich erstaunlich rasch der neuen Situation an - ihre europäischen Mitglieder fochten zusammen mit den USA und weiteren Partnern einen langen, verlustreichen Krieg, der bis heute anhält. Das endgültige Urteil über das Engagement am Hindukusch steht noch aus. Entscheidend ist, dass die NATO ohne ihre Fähigkeit zur Anpassung an neue sicherheitspolitische Lagen ihre Bedeutung bereits mit dem Fall der Berliner Mauer weitgehend eingebüßt hätte. Eine solche Lageänderung zeichnet sich ab und das Bündnis wird sich auch auf diese Situation einstellen müssen, wenn es weiterhin das erfolgreichste Sicherheitsbündnis der neueren Geschichte bleiben will. Mehr noch, würde die NATO in ihrem Wert ausgehöhlt, so würde Verteidigungspolitik wieder allein in die Hand der Nationalstaaten fallen. An einer solchen militärischen ReNationalisierung Europas kann weder die eine noch die andere Seite des Atlantiks ein Interesse haben.

\section{Literatur}

Binnendijk, Hans Hrsg. (2014): The Transatlantic Pivot to Asia. Washington D.C.: Brookings Institution.

Brauss, Heinrich (2018): Nato Beyond 70. Renewing a Culture of Readiness. Tallinn: International Centre for Defence and Security, https://icds.ee/wp-content/uploads/2018/11/ ICDS-Analysis_NATO-Beyond-70_Heinrich-Brauss_November2018-1.pdf.

Rühle, Michael (2006): Entwicklungslinien des Atlantischen Bündnisses, APUZ 43, Bundeszentrale für politische Bildung: Bonn, http://www.bpb.de/apuz/29454/entwicklungsliniendes-atlantischen-buendnisses? $\mathrm{p}=$ all.

Towell, Pat/Kazlauskas, Aras D. (2018): The European Defense Initiative: a budgetary overview. Washington, D.C.:, Congressional Research Service. 